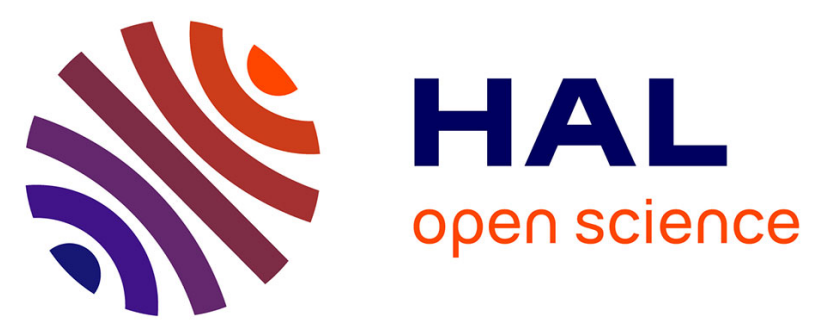

\title{
Temperature influence on pH-ISFET sensor operating in weak and moderate inversion regime: Model and circuitry
}

Salah Edine Naimi, Bekkay Hajji, Iryna Humenyuk, Jérôme Launay, Pierre Temple-Boyer

\section{To cite this version:}

Salah Edine Naimi, Bekkay Hajji, Iryna Humenyuk, Jérôme Launay, Pierre Temple-Boyer. Temperature influence on pH-ISFET sensor operating in weak and moderate inversion regime: Model and circuitry. Sensors and Actuators B: Chemical, 2014, 202, pp.1019 - 1027. 10.1016/j.snb.2014.06.008 . hal-01504988

\section{HAL Id: hal-01504988 \\ https://hal.science/hal-01504988}

Submitted on 10 Apr 2017

HAL is a multi-disciplinary open access archive for the deposit and dissemination of scientific research documents, whether they are published or not. The documents may come from teaching and research institutions in France or abroad, or from public or private research centers.
L'archive ouverte pluridisciplinaire HAL, est destinée au dépôt et à la diffusion de documents scientifiques de niveau recherche, publiés ou non, émanant des établissements d'enseignement et de recherche français ou étrangers, des laboratoires publics ou privés. 


\title{
Temperature Influence on pH-ISFET sensor operating in Weak and Moderate Inversion Regime : Model and circuitry
}

\author{
S. E. Naimi ${ }^{\mathrm{a}, *}$, B. Hajji ${ }^{\mathrm{a}}$, I. Humenyuk ${ }^{\mathrm{b}, \mathrm{c}}$, J. Launay ${ }^{\mathrm{b}, \mathrm{c}}$, P. Temple-Boyer ${ }^{\mathrm{b}, \mathrm{c}}$ \\ a "SDMN Team", National School of Applied Sciences, BP 669, Oujda-60000, Morocco \\ ${ }^{b}$ CNRS, LAAS, 7 avenue du colonel Roche, F-31400 Toulouse, France \\ ${ }^{c}$ University of Toulouse; UPS; LAAS; F-31400 Toulouse, France
}

\begin{abstract}
Usually most pH-ISFET readout circuits, with temperature compensation, were designed using transistors operating in strong regime. However, a classes of circuits elaborated with respect to MOS weak inversion are also very suitable for low-voltage and low-power applications. In this work, we discuss the problem of temperature variation at the sensor and circuit level. An analysis was made of the sensor operating in weak and moderate inversion regime. It has been shown that a simplified version of the EKV model combined with site-binding model can describe the behavior of ISFET toward the temperature and $\mathrm{pH}$ change. The experimental results agree very well with the analytical model for devices in large intervals of $\mathrm{pH}$ and temperature. Finally, the usage of model development is considered with an original concept of a readout circuit. The result of the simulation shows that the output signal is linear with $\mathrm{pH}$, the design technique permits improving temperature insensitivity. The proposed circuit can be integrated with an ISFET by standard CMOS technology.
\end{abstract}

Keywords: pH-ISFET, EKV-model, Weak inversion, Thermal drift, Readout circuit

\section{Introduction}

Since its development in the 1970 [1], the Ion-Sensitive field effect transistors (ISFET) has been receiving more attention especially in areas of environmental monitoring applications and biomedical analysis. As a result, it has been used for the detection of multiple ions due to its many ion-sensitive membranes $[2,3]$. However, many industrial and laboratory applications require the precise measurements of $\mathrm{pH}$. Commercially available sensors are generally very expensive devices, especially if reasonable precision and reliability are requested. Several investigations have demonstrated that the pH-ISFET sensor presents some drawbacks related to thermal dependency and long-term drift [4-6]. These factors limit the performances of pH-ISFET sensors, leading to unacceptable results in critical measurements. In order to improve the pH-ISFETs stability, a lot of temperature compensation techniques have been reported in the literature such as the proper choice of biasing current for isothermal operating point to reduce ISFET temperature coefficient, the use of a $\mathrm{p}-\mathrm{n}$ junction diode for temperature compensation on a single ISFET device, an ISFET based operational amplifier employment that improves temperature stability or the combination of several methods [7-9]. At the same time a lot of work has been done in the last thirty years, including simulations of its temperature characteristics according to theoretical models. They show that the ISFET temperature dependence is complicated in nature. Generally, these models were based

\footnotetext{
${ }^{*}$ Corresponding author. Tel: +212-536-505-470; Fax: +212-536-505-471

Email address: snaimi@ensa. ump.ma (S. E. Naimi)
}

on the so-called site-binding model and the MOSFET theory. The temperature influence is relatively complicated since it's related to the reference electrode, electrolyte/insulator potential and ISFET based MOS transistors [10]. Additionally, the ISFET sensor is used, classically, in his strong inversion regime, this implies high power consumption, while the trend towards low-voltage operation, both for reasons of compatibility with digital technology, and to meet the needs of battery-operated equipment. Another strategy is to consider the weak-inversion operating. Previous studies have shown this possibility [11]. Sub-threshold MOS operation, or weak inversion, is most often required by the low-power applications in modern electronics.

This article reviews our recent successful modeling of the temperature influence on pH-ISFET sensors by using MOSFET theory and site-binding model. In this work we implemented a simplified Enz-Krummenacher-Vittoz (EKV) model to explain the characteristics of ISFET sensor [12], in weak and moderate inversion regime, and discuss how the temperature can affect the behavior of the sensor. This paper deals with temperaturedependent ISFET drift modeling development and to its validation by comparison with experimental measurements. We have, also, investigated an alternative circuit operating in a subthreshold regime to have a linear relation between an output and the $\mathrm{pH}$, and an improved thermal stability of this output.

\section{ISFET electrical properties : background review}

\section{1. $p H$ sensitive film/electrolyte interface modeling}

Figure 1 shows a simplified schematic representation of the pH-ISFET sensor using p-type silicon substrates. The basic 


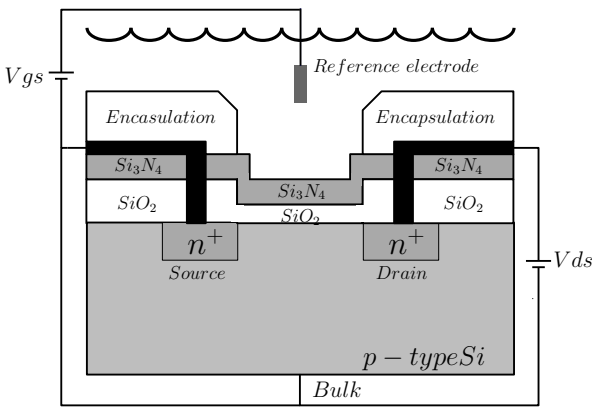

Figure 1: Schematic of the pH-ISFET structure (N-Type).

structure that serves as the basis for the ISFET is fabricated in standard CMOS chip technology. In the following and based on already existing theories, we combined the well-known site binding model and the MOSFET model to describe the behavior of an ISFET sensor. In many devices of this kind, it is assumed that protons interact with the insulator according to sitebinding theory. Whereas in the case of silicon nitride membrane, several studies have demonstrated that when the $S i_{3} N_{4}$ surface is exposed to water, a sensitive surface becomes oxygen rich [13]. Consequently the silicon nitride surface contains sites in two forms, silanol $(\mathrm{SiOH})$ and amine groups $\left(\mathrm{SiNH}_{2}\right)$, previous works have mentioned that all other sites can be neglected. More details on the site-binding theory can be found in [14-16]. A first-order model simplification suggests that if the electrolyte concentration is reasonable, the hypothesis of mono-sites is plausible. With this simplification the response of the sensor to the $\mathrm{pH}$ is given by the surface potential $\psi_{0}$ and dependent on the $\mathrm{pH}$ value of the electrolyte, it can be expressed as:

$$
\psi_{0}=2.303 \frac{K T}{q} \frac{\beta_{0}}{1+\beta_{0}}\left(p H_{p z c}-p H\right)
$$

where $T$ is the temperature of the system, $K$ is the Boltzmann's constant, $q$ the electron charge, $p H_{p z c}$ is the non zero $\mathrm{pH}$, and $\beta_{0}$ is a parameter which reflects the chemical sensitivity of the gate insulator and is dependent on the density of surface sites $N s$ and the double layer capacitance $C_{D L}$. The parameter $\beta_{0}$ is given by:

$$
\beta_{0}(T)=\frac{2 q^{2} N s \sqrt{K a \cdot K b}}{K T C_{D L}}
$$

where $K a, K b$ are dissociation constants for the chemical reactions at the insulator interface. The double layer capacitance $C_{D L}$ consists of the two series capacitances $C_{\text {Gouy }}$ and $C_{H e l m}$, where $C_{\text {Gouy }}$ represent the equivalent Gouy-Chapman capacitance and $C_{H e l m}$ the Helmholtz capacitance, which has already been developed by site-binding theory and the electrical doublelayer theory $[17,18]$. It should be noted that $C_{\text {Gouy }}$ and $C_{H e l m}$ are temperature dependent, however, since these are secondorder effects, we will assume $C_{\text {Gouy }}$ and $C_{H e l m}$ to be independent of temperature [5].

Thus, it has been possible to investigate the pH-ISFET flatband voltage shift with $\mathrm{pH}$ and the variation of the potential at

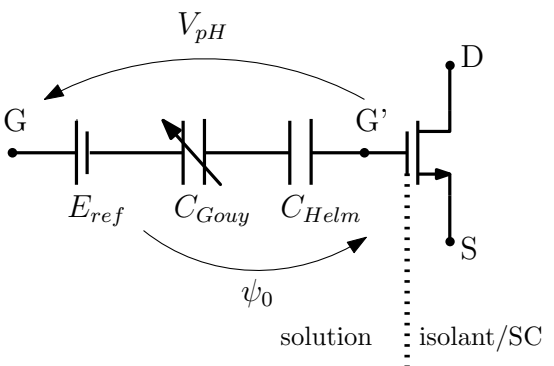

Figure 2: Equivalent circuit for the pH-ISFET macro-model

the electrolyte/gate dielectric interface. In summary, the flatband voltage shift has a standard expression:

$$
\Delta V_{F B}=V_{p H}=E_{r e f}+\chi^{s o l}+\Delta \varphi^{l j}-\psi_{0}
$$

where $E_{\text {ref }}$ is the potential of $\mathrm{Ag} / \mathrm{AgCl}$ reference electrode, $\Delta \varphi^{l j}$ is the potential drop between the reference electrode and the solution, which typically has a value of $3 \mathrm{mV}, \chi^{\text {sol }}$ is the electrolyte-insulator surface dipole potential, with a typical value of $50 \mathrm{mV}$ [10]. Both $\Delta \varphi^{l j}$ and $\chi^{\text {sol }}$ are $\mathrm{pH}$ independent. $\psi_{0}$ is the surface potential from (1). In equation (3), $\psi_{0}$ is the only parameter responsible for ISFET $\mathrm{pH}$ sensitivity, as previously explained by the site binding theory. Hence the macro-model represented schematically in Fig.2.

\subsection{MOSFET part modeling : EKV Model}

The working principle of the ISFET is based on the standard MOSFET structure. Therefore, a robust model, of the MOSFET part, is suitable for performing robust and valid model in a large interval of temperatures and $\mathrm{pH}$. Another critical point might be the number of parameters for each model, who must be smallest. EKV model is particularly suitable as it provides accurate modeling with a small set of parameters [12]. This section describes the equations and parameters used for the computer simulation version of the EKV-MOSFET model. The set of equations includes modeling of basic physical effects, geometrical aspect, substrate effect, modeling of mobility reduction due to vertical field and modeling of thermal behavior. The EKV model proposes the following empirical expression of source-drain current $\left(I_{D S}\right)$ valid for all regimes of operating.

$$
I_{D S}=2 n \beta U_{T}^{2}\left[\ln ^{2}\left(1+e^{\frac{V_{G S}-V_{T 0}-n . V_{S}}{2 n U_{T}}}\right)-\ln ^{2}\left(1+e^{\frac{V_{G S}-V_{T 0}-n . V_{D}}{2 n U_{T}}}\right)\right](4)
$$

where $U_{T}$ is the thermodynamic voltage, $V_{T 0}$ is the threshold voltage, $n$ is the body effect factor and $\beta$ the transfer parameter, $V_{G S}$ is the gate to source voltage, $V_{S}$ is the source potential and $V_{D}$ is the drain potential. For long channel model implementation, we consider only parameters accounting for first order effects. The model used is based on the ideal MOS structure of long and large channel with uniform substrate doping. Notice that the current expression in (4) is let as a difference between forward and reverse current using standard interpolation function, thus a better fitting is obtained in weak and moderate inversion. The expressions of EKV parameters $V_{T 0}, n$ and $\beta$ are: 


$$
\begin{aligned}
& V_{T 0}=V_{F B}+\phi_{F}+\gamma \sqrt{\phi_{F}} \\
& n=1+\frac{\gamma}{2 \sqrt{V_{P}+\phi_{F}+4 U_{T}}} \\
& \beta=K_{P} \frac{W}{L} \frac{1}{1+\theta \cdot V_{P}}
\end{aligned}
$$

where $V_{F B}$ is the flat-band voltage, $\phi_{F}$ is the bulk Fermi potential, $\gamma$ is the body effect parameter, $K_{P}$ is the transconductance parameter, $W$ is the channel width and $L$ is the channel length. The mobility reduction modeling introduces only one parameter, $\theta$. Finally $V_{P}$ is the pinch-off voltage.

Basically, $V_{P}$ and $n$ factors are specific to EKV model, although a possible physical link between EKV model and charge-sheet model seem to exist [19]. The $V_{P}$ term expression is:

$$
V_{P}=V_{G S}-V_{F B}-\phi_{F}-\gamma\left(\sqrt{V_{G S}-V_{F B}+\left(\frac{\gamma}{2}\right)^{2}}-\frac{\gamma}{2}\right)
$$

\section{ISFET Temperature characteristic}

It seems that the pH-ISFET sensor, sometimes, do not follow the theory of operation. The data obtained show that the temperature dependence is complicated in nature and the user should beware of using the sensors during variable environmental conditions e.g. field tests. In such case an additional electronic circuit is needed to reduce the temperature drift of the sensor response. In order to explore a pH-ISFET parameters, subject to temperature changes, this section describe a simplified temperature-dependent model of MOSFET part of the $\mathrm{pH}$ ISFET as well as the reference electrode temperature influence. We show, later, that these compact models provide accurate representation of measured DC component performance.

\subsection{MOSFET temperature effect}

The EKV model introduces a few parameters for intrinsic parameter's temperature dependence, we consider two of them here, TCV the temperature coefficient of the threshold voltage and BEX the mobility temperature exponent [20].

$$
\begin{aligned}
& V_{T 0}(T)=V_{T 0}-T C V\left(T-T_{\text {nom }}\right) \\
& K_{P}(T)=K_{P}\left(\frac{T}{T_{\text {nom }}}\right)^{B E X}
\end{aligned}
$$

The temperature dependence of the bulk Fermi potential $\phi_{F}$ is as :

$$
\begin{gathered}
\phi_{F}(T)=\phi_{F} \frac{T}{T_{\text {nom }}}-3 \frac{K T}{q} \ln \left(\frac{T}{T_{\text {nom }}}\right) \\
-E g\left(T_{\text {nom }}\right) \frac{T}{T_{\text {nom }}}+\operatorname{Eg}(T)
\end{gathered}
$$

Where $E g$ is the energy gap of silicon, which should obey :

$$
E g(T)=1.16-0.000702 \cdot \frac{T^{2}}{T+1108}
$$

Where $T$ is the absolute temperature and $T_{n o m}$ is the nominal temperature chosen as a reference temperature $\left(T_{n o m}=\right.$ $300.15 C$ ). The intrinsic model parameters $\gamma, \phi_{F}$ and $K_{P}$ are related to process parameters like oxide thickness and channel doping. Generally it is preferable to avoid to link such parameters for better fitting of experimental measurements.

\subsection{Temperature coefficient of the reference electrode}

The liquid solution, which acts as the gate ion-conductor, is connected to the gate voltage source by means of an $\mathrm{Ag} / \mathrm{AgCl}$ reference electrode. The potential of the reference electrode $E_{r e f}$ is related to the temperature according to the expression [10]:

$$
\begin{gathered}
E_{r e f}(T)=E_{a b s}\left(\frac{H^{+}}{H_{2}}\right)+E_{r e f}\left(\frac{A g}{A g C l}\right) \\
+\left(\frac{d E_{r e f}}{d T}\right)(T-298.16)
\end{gathered}
$$

Where $E_{a b s}\left(H^{+} / H_{2}\right)$ is the normalized hydrogen potential, $E_{r e f}(\mathrm{Ag} / \mathrm{AgCl})$ is the relative potential of the reference electrode, the two potential are temperature independent (their values are respectively $4.7 \mathrm{~V}$ and $0.205 \mathrm{~V}$ ), the temperature coefficient of $\mathrm{AgCl} / \mathrm{Solution}$ junction is practically negligible, $d\left(E_{r e f}\right) / d T$ is the temperature coefficient equal to $140 \mu \mathrm{V} / \mathrm{K}$ [21-23].

\section{Fabrication and testing}

The N-type ISFET fabrication was carried out at LAAS/CNRS clean room facilities. Standard N+ implantation (dopant: arsenic, energy: $50 \mathrm{keV}$, dose: $10^{16} \mathrm{at} / \mathrm{cm}^{2}$ ) was used to realize the source and drain $\mathrm{N}+$ regions into the boron-doped $\left(10^{15} \mathrm{at} / \mathrm{cm}^{3}\right)$ P-type silicon substrate. The gate dielectric is based on a $\mathrm{SiO}_{2} / \mathrm{Si}_{3} \mathrm{~N}_{4}$ sensitive membrane structure [24]. It is obtained by a thermal oxidation, in dry oxygen, to grow a thin layer of $\mathrm{SiO}_{2}$ (thickness: $50 \mathrm{~nm}$ ) followed by a low pressure chemical vapor deposited (LPCVD) process to form on top a thin $\mathrm{Si}_{3} \mathrm{~N}_{4} / \mathrm{SiO}_{2}$ film (thickness: 50nm) acting as ion-sensitive layer as well as wafer-level passivation layer. The length of the canal is $30 \mu \mathrm{m}$ and its width is $800 \mu \mathrm{m}$. To measure the current-voltage characteristics of the pH-ISFET, a source and substrate are grounded and the source to drain voltage was fixed to $1 \mathrm{~V}$. To measure $\mathrm{pH}$ sensitivity, the $\mathrm{pH}$ buffer solutions were changed from $\mathrm{pH} 2$ to $\mathrm{pH} 10$. To investigate the stability and reliability of the $\mathrm{pH}$ sensors, the effects of temperature on the responses of an ISFET are evaluated.

Temperature can change the characteristics of a sensor, the potential of a reference electrode, the $\mathrm{pH}$ of electrolyte and the whole measuring system. For latter changes, constant temperature is maintained inside the room. To keep the electrode, electrolyte, ISFET temperature constant, a heat regulating loop including a temperature-sensing device and a heating device have been used. Also, experimental results can only be compared validly to theory, if the temperature during measurements is fixed. We use a temperature controller to set the temperature in the range of $23-50^{\circ} \mathrm{C}$. 


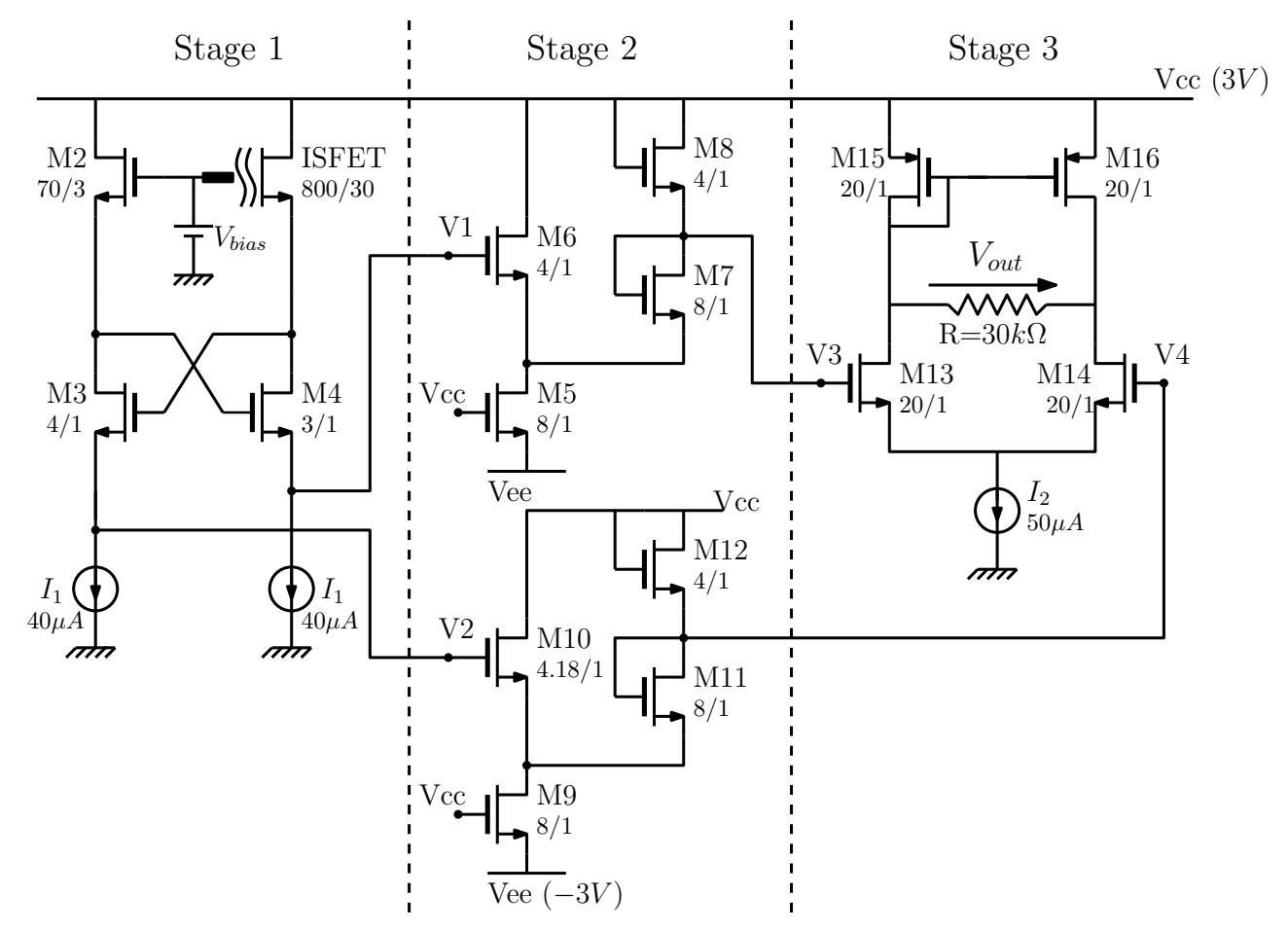

Figure 3: Proposed readout circuit to provide high thermal stability and linearity, stage 1: Caprio's quad, stage 2 : attenuators, stage 3 : differential amplifier. The ratio values indicate $\mathrm{W} / \mathrm{L}$ in $\mu \mathrm{m}$. The potential Vbias is set to $V_{C C}$ (it may vary from $2.5 \mathrm{~V}$ to $3 \mathrm{~V}$ ). The transistor M2 is used in conjunction with the transistor M4 to control the variation of $V_{1}$, thus the ration $70 / 3$ of M2 don't match the ration 800/30 of the pH-ISFET.

\section{The readout circuit}

Since the purpose of this work is to study the benefit of using an ISFET sensor in weak-inversion regime, we propose a simple readout IC with low temperature dependence. From (4) it can be easily shown that the source-drain current $I_{D S}$, in weak inversion region, must comply with the following requirement $\left(V_{S}=0, V_{D S} \gg V_{T}\right)$ :

$$
I_{D S}=2 n \beta U_{T}^{2} e^{\frac{V_{G S}-V_{T 0}-V_{p H}}{n U_{T}}}
$$

With $V_{p H}=\Delta V_{F B}$, thus the macro-model of the ISFET in Fig. 2 is still available in weak inversion. This section explores some ideas on how to use such results in a simple circuit.

\subsection{Basic principle of the circuit}

For $V_{p H}$ we get from equations (1)(3) and (13):

$$
\begin{aligned}
V_{p H}= & 2.303 U_{T}\left(p H-p H_{p z c}\right)+E_{r e f}\left(\frac{A g}{A g C l}\right) \\
& +\left(\frac{d E_{r e f}}{d T}\right)(T-298.16)+\chi^{s o l}+\Delta \varphi^{l j}
\end{aligned}
$$

The absolute potential of the standard hydrogen electrode $E_{a b s}\left(\frac{H^{+}}{H_{2}}\right)$ is compensated by the work function of the metal back contact/electronic charge [18]. Let us consider the expression in (15), it can be rewritten as:

$$
V_{p H}=V_{1}-V_{2}=a_{p H} U_{T}+b
$$

With

$$
\begin{cases}a_{p H} & =2.303\left(p H-p H_{p z c}\right)+\frac{q}{k} \frac{d E_{r e f}}{d T} \\ b & =E_{r e f}\left(\frac{A g}{A g C l}\right)-298.16 \frac{d E_{r e f}}{d T}+\chi^{s o l}+\Delta \varphi^{l j}\end{cases}
$$

These two terms, $a_{p H}$ and $b$, being to the first approximation independent of the temperature. The temperature-dependency of the first term of $V_{p H}\left(a_{p H} U_{T}\right)$ can be eliminated if the pHISFET is operating in weak inversion. The problem, however, will still not be solved; the resulting term $b / U_{T}$ cannot be eliminated easily (as by a differential structure). In the following analysis, we focus on this second term and try to minimize its influence. In equation (16), $V_{p H}$ is expressed as a differential potential $\left(V_{1}-V_{2}\right)$, if each potential passes through an attenuator circuit, the output of the attenuator stage is $\left(V_{3}-V_{4}\right)$, with:

$$
V_{3}-V_{4}=\alpha_{1} V_{1}-\alpha_{2} V_{2}
$$

yielding

$$
\frac{V_{3}-V_{4}}{U_{T}}=\frac{\alpha_{1} V_{1}-\alpha_{2}\left(V_{1}-b\right)}{U_{T}}+\alpha_{2} a_{p H}
$$

If $\alpha_{1}$ and $\alpha_{2}$ are chosen as $\alpha_{1} V_{1} \approx \alpha_{2}\left(V_{1}-b\right)$, the expression in equation 19 becomes :

$$
\frac{V_{3}-V_{4}}{U_{T}} \approx \alpha_{2} a_{p H}=\alpha_{2}\left(2.303\left(p H-p H_{p z c}\right)+\frac{q}{k} \frac{d E_{r e f}}{d T}\right)(20)
$$

The last expression in equation (20) is a function of $\mathrm{pH}$ but temperature independent (we suppose that the effect of the temperature on $p H_{p z c}$ and $d E_{r e f} / d T$ is negligible). However, the 
term $\alpha_{2}$ must be temperature independent. To achieve the earlier condition, $\alpha_{1} V_{1} \approx \alpha_{2}\left(V_{1}-b\right)$, the potential $V_{1}$ does not have to vary too much. In the next subsections we propose a circuit able to extract the potential $V_{p H}$ and then divide it by the thermal potential $U_{T}$. This illustrates the general principle of the proposed circuit. The basic topology of this circuit is given in Fig. 3, it has three stages as shown in the figure. As suggested previously, once the tension $V_{p H}$ is extracted (with the first stage), the two components of a differential potential are passed through two attenuators in the second stage, the third stage allows to divide the output by a thermal voltage $U_{T}$. In the previous considerations, the voltage $V_{1}$, can obviously be fixed by a reference voltage, placed in the gate of the transistor M13. However, this tension is used actually to keep for each stage, the tension resulting from the sensor as a differential voltage. This will allow a better linearization of the output.

\subsection{First stage : modified Caprio's Quad}

The first stage of the circuit in Fig. 3 is based on a Caprio's Quad, which is originally a V-I converter. The circuit is useful for moderate input amplitudes, the voltage swing at the input cannot be very large $( \pm 500 \mathrm{mV}$ at most $)$. The equivalent voltage source $V_{p H}$ is accurately replicated across the source connections of M3 and M4. The current sources $I_{1}$ allow limiting the currents in a desired region (sub-threshold or moderate inversion regions). Obviously the $\mathrm{pH}$-ISFET and the transistor M2 should have a perfectly matched ration $W / L$. As previously mentioned, the potential $V_{1}$ must be constant or variable in reasonable limits, this can be achieved by adjusting the width of the transistor M4. Consequently the greatest variation of the voltage $V_{1}$ was found to be around $6 \%$ for large variations of the temperature and the $\mathrm{pH}\left(T=20-60^{\circ} \mathrm{C}, \mathrm{pH}=2-12\right)$. Thus, the voltage swing, $V_{2}-V_{1}$ is caused by the variation of $V_{2}$. In the next section we will discuss this results.

\subsection{Second stage : attenuators}

The output of each attenuator (M5, M6, M7, M8 or M9, M10, $\mathrm{M} 11, \mathrm{M} 12)$ is linearly proportional to inputs. One of these attenuators can be used to have $\alpha_{1} V_{1} \approx \alpha_{2}\left(V_{1}-b\right)$ as mentioned previously. In fact we need this stage to reduce the potentials values for the entry of the third stage too. It is assumed that good layout techniques will help reduce errors due to process and thermal variations. These circuits are very immune to temperature for great variations of the entry.

\subsection{Third stage : source coupled circuit}

A differential amplifier composed of the two matched transistors M13 and M14 in addition to the source current $I_{2}$, provides a simple method of realizing a division of the input voltage by the thermal potential $U_{T}$. The basic equation of the circuit is :

$$
I_{\text {out }}=I_{D M 13}-I_{D M 14}=I_{2} \times \tanh \left(\frac{V_{d}}{2 \eta U_{T}}\right)
$$

where $I_{D M 13}, I_{D M 14}$ are the drains current of M13 and M14, $V_{d}$ is the differential input voltage $\left(V_{d}=V_{3}-V_{4}\right)$ and $\eta$ is the sub-threshold slope factor. Equation (21) assumes that M13 and
M14 are operating in weak inversion. If the circuit operates under the restriction, $\left|V_{d}\right|<<U_{T}$, the output current of the circuit is given by :

$$
I_{\text {out }} \approx I_{2} \frac{V_{d}}{2 \eta U_{T}}
$$

The circuit uses the current mirror as an active load (M15M16). Note that the output of the circuit is a current, and if it is required to have an output voltage, a differential current to voltage converter is needed. To simplify this can be realized by using a resistor $(R)$ as shown, in this case, the output voltage is given by:

$$
V_{\text {out }} \approx R I_{2} \frac{V_{d}}{4 \eta U_{T}}
$$

As the differential-pair (M13-M14) does not operate correctly for large signal, therefore we are constrained to make the Caprio's quad signals pass through two attenuator circuits to reduce the input voltages. In order to demonstrate the feasibility of the proposed circuit and to show an improvement of the temperature independent output, we simulate this circuit by using the MOSIS CMOS (AMI 1.0 $\mu \mathrm{m}$ ) minimum channel length technology and SPICE parameters. The electronic circuit simulation program HSPICE, which is one of the simulators that can be suitably adapted to design and simulate the ISFET transducers has been used.

\section{Simulation results and discussion}

\subsection{ISFET characteristics}

The equations provided in the previous sections are used to simulate the ISFET behavior under different operating conditions. The EKV parameters and site-binding coefficients are determined from experimental data. In order to obtain an optimized set of coefficients, it is desirable to take into account all types of experimental data, e.g. temperature, $\mathrm{pH}$ and gatesource voltage $(V g s)$ changes. The parameters are determined from the experimental data by an efficient mathematical, least squares minimization, methods.

Drain to source current versus gate to source voltage (Ids $V g s)$ curves, measured in buffer solutions of different $\mathrm{pH}$, are shown in Fig. 4-5-6. We present the results as a series of plots. The curves are shifted by $54.7 \mathrm{mV} / \mathrm{pH}$ at $23^{\circ} \mathrm{C}$ to a positive voltage for the samples immersed in higher-pH buffer solutions, which can be explained by the site binding theory. The experimental results were found to be in agreement with the theoretical predictions. As can be seen from Fig.7, the fitted curves accurately match the measured values at $\mathrm{pH} 4,10$ and specific value of pH 7 (Fig.9-b). In a second experiment, the pH-ISFET was operating in a buffer solution at $\mathrm{pH} 5.2$ with temperature varied in from 10 to $52^{\circ} \mathrm{C}$. Taking into account that the model is relatively simple, the simulation results are satisfactory. The fit is excellent, for all ranges of $\mathrm{pH}$ and temperature in weak inversion and remain good for moderate inversion. Obviously, for long and wide device we would expect the best agreement, with a few MOSFET part parameters. 


\begin{tabular}{lllll}
\hline & \multicolumn{2}{c}{$\begin{array}{c}\text { Process I } \\
N_{A} \approx 1 e^{17}\left(\mathrm{~cm}^{-3}\right)\end{array}$} & \multicolumn{2}{c}{ Process II } \\
& \multicolumn{1}{c}{$N_{A} \approx 1 e^{15}\left(\mathrm{~cm}^{-3}\right)$} \\
\hline Parameters & Mean Values & Standard deviation & Mean Values & Standard deviation \\
\hline$\gamma(\sqrt{V})$ & 6.0147 & 1.3297 & 3.0661 & 0.7800 \\
$\phi_{F}(V)$ & 0.5318 & 0.2682 & 0.4630 & 0.1704 \\
$K_{P}\left(A / V^{2}\right)$ & $5.8406 \times 10^{-4}$ & $5.7192 \times 10^{-4}$ & $12 \times 10^{-4}$ & $4.6700 \times 10^{-4}$ \\
$\theta(1 / V)$ & 2.5076 & 1.1121 & 25.8612 & 5.7460 \\
$V_{T 0}(V)$ & 0.4160 & 0.1413 & -0.6401 & 0.2660 \\
$B E X$ & -1.0914 & 0.0274 & $(*)$ & - \\
$T C V(V / K)$ & $8.6 \times 10^{-3}$ & 0.0150 & $(*)$ & - \\
$p H_{p z c}$ & 4.2389 & 0.3364 & 4.1223 & 0.2168 \\
$\beta_{0} \times U_{T}(V)$ & 0.2370 & 0.0032 & 0.2209 & 0.0045 \\
\hline$R F$ & 0.0294 & 0.0129 & 0.0319 & 0.0083 \\
\hline
\end{tabular}

Table 1: Extracted model parameters for MOSFET part and surface potential parameters values with regression statistics for a total of 23 tested devices. $\left(N_{A}\right.$ is the substrate p-type doping concentration, $R F$ is the Reliability Factor (RF was calculated for each device), (*) no temperature variations were made for this process).

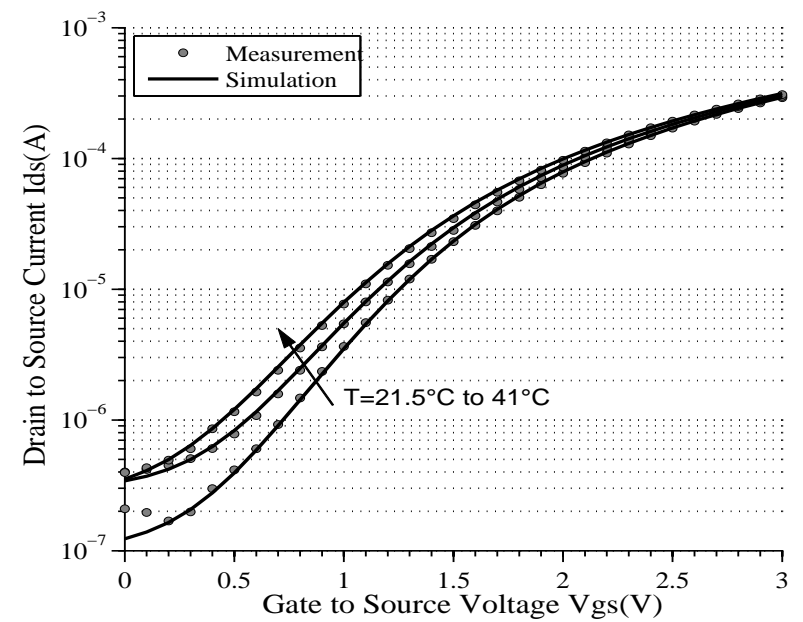

Figure 4: Drain-Source current $(I d s)$ vs Gate-Source voltage $(V g s)$ for $p H=2$, $T=21.5^{\circ} \mathrm{C}-31^{\circ} \mathrm{C}-41^{\circ} \mathrm{C}$

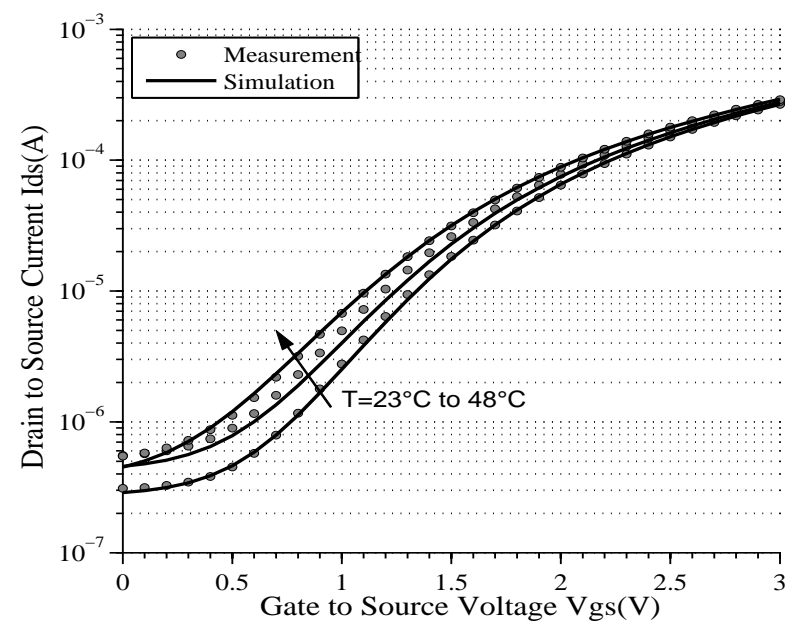

Figure 5: Drain-Source current ( $I d s)$ vs Gate-Source voltage $(V g s)$ for $p H=4$, $T=23^{\circ} \mathrm{C}-33^{\circ} \mathrm{C}-48^{\circ} \mathrm{C}$
The model parameters (EKV and site binding) were obtained through a nonlinear regression process, by the least squares method. The mean value, as well as the standard deviation of each parameter are given in table 1. Data processing was done for 23 devices tested under various $\mathrm{pH}$ and temperature conditions. As we used a nonlinear regression, the reliability factor (RF) defined in equation 24 is used to measure of the goodness of fit instead of the coefficient of determination $\left(R^{2}\right)$. In equation $24, I d s_{\exp }(i)$ is the experimental measurement number i, while $I d s_{t h}(i)$ is the calculated values.

$$
\text { Reliability_Factor }=\sqrt{\left(\frac{\sum_{i=1}^{n}\left(I d s_{\text {exp }}(i)-I d s_{t h}(i)\right)^{2}}{\sum_{i=1}^{n}\left(I d s_{\text {exp }}(i)\right)^{2}}\right)}
$$

\subsection{Temporal drift and hysteresis experiments}

Drift phenomena in $\mathrm{pH}$-ISFETs typically appear as relatively slow, monotonic, and temporal changes in the threshold voltage of the device. Figure 8 shows the gate to source $(V g s)$ voltage drift when $I d s$ and $V d s$ were fixed to $100 \mu \mathrm{A}$ and $1 \mathrm{~V}$ respectively, while the buffer solution is at $\mathrm{pH} 10$. The test was made for approximately $4 \mathrm{~h}$. A heating/cooling cycle has been performed to estimate the influence of the temperature on the $\mathrm{pH}$ ISFET. The experiment of the illumination influence has been performed in the same test (by switching on the light, the room illumination has been found to increase from 70 to $1500 \mathrm{Lux}$ ). The shift of $\Delta V g s$ is around $5 m V$ (equivalent to $0.1 \mathrm{pH}$ measurement error). Firstly, the pH-ISFETs responses are characterized by a decrease which should be related to their adaptation to the watery medium. The increase of temperature from $22^{\circ} \mathrm{C}$ to $40^{\circ} \mathrm{C}$ is responsible for an increase of the output voltage around $25 \mathrm{mV}$. This phenomenon is related to the decrease of the ISFET threshold voltage. The initial period being apart, the complete experiment has also allowed estimating the temporal drift of the pH-ISFETs around 7.5mV/hour, i.e. $0.15 \mathrm{pH} /$ hour. When the heating/cooling cycle is no longer, the temporal drift is still evidenced but is strongly reduced. The drift can be estimated to $1 \mathrm{mV} /$ hour, i.e. $0.02 \mathrm{pH} /$ hour. Like the time drift effect, the hysteresis also limits the accuracy of ISFET pH measurements. Hysteresis tests were performed by varying the $\mathrm{pH}$ 


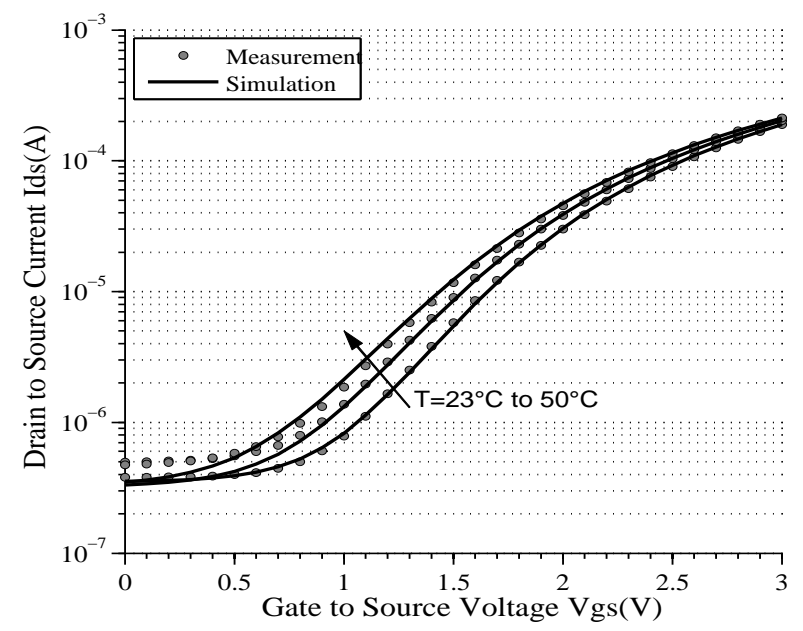

Figure 6: Drain-Source current $(I d s)$ vs Gate-Source voltage $(V g s)$ for $p H=$ $10, T=23^{\circ} \mathrm{C}-37^{\circ} \mathrm{C}-50^{\circ} \mathrm{C}$

to close a loop $(4-7-10 \leftrightarrow 10-4-7)$. All tested devices exhibit hysteresis of approximately $8-10 \mathrm{mV}$, in sub-threshold region. Figure 9 depicts the hysteresis curves measured under the $\mathrm{pH}$ loop at $T=22^{\circ} \mathrm{C}$ and $\mathrm{Id} s=20 \mu \mathrm{A}$.

All series of measurements have been devoted to characterizing the static sensor response, i.e. the dependence of the sourcedrain current versus source-grid tension on the $\mathrm{pH}$ and temperature changes in weak and moderate inversion regime. Based on these results, the behavioural thermal drift of the $\mathrm{pH}$-ISFET in the sub-threshold region can be modeled by a combination of basic EKV model for the MOSFET part of the ISFET and a simplistic version of the site-binding model using Martinoia macro-model [25] presented in Fig. 2. On the other hand, the electrolyte-membrane part plays an important role in the static characteristics of the sensors. The thermal effect of the electrode/electrolyte/membrane cannot be neglected and limiting the performance of the sensor $\left(E_{r e f}\right.$ drift is around $0.14 m V /{ }^{\circ} \mathrm{C}$, the threshold voltage thermal drift of the MOS part is around $9 m V /{ }^{\circ} \mathrm{C}$ and the surface potential drift is around $1 \mathrm{mV} /{ }^{\circ} \mathrm{C}$ ).

\subsection{Circuit simulation}

Figure 10 shows the temperature and the $\mathrm{pH}$ actions on the first stage outputs $\left(V_{1}\right.$ and $\left.V_{2}\right)$. As can be seen from the figure, the $\mathrm{pH}$ influences significantly the potential $V_{2}$ instead of the potential $V_{1}$. The maximal variation of $V_{1}$ can be estimated around $19 \mathrm{mV}(6 \%)$, while that of $V_{2}$ is approximately $410 \mathrm{mV}$, for $\mathrm{pH}$ varying from 2 to 12 . As an analytical calculation of the attenuation $\alpha_{1}\left(\alpha_{2}\right)$ is very difficult (especially to take into account of the effect of the temperature variation). The influence of the temperature, the canal width of the transistor M10 and the $\mathrm{pH}$ on the terms $\alpha_{1} V_{1}, \alpha_{2}\left(V_{1}-b\right)$ and $\alpha_{1} V_{1}-\alpha_{2}\left(V_{1}-b\right)$ was simulated. The results of these simulations are shown in figure 11. The circuit displayed a minimum change of the term $\alpha_{1} V_{1}-\alpha_{2}\left(V_{1}-b\right)$ under several conditions such as the change of temperature or the potential $V_{1}$. In figure 11 the change of the potential $V_{1}$ is imposed by the variation of the $\mathrm{pH}$ from 1 to 12 , the potential $V_{1}$ was extracted from the simulation of the
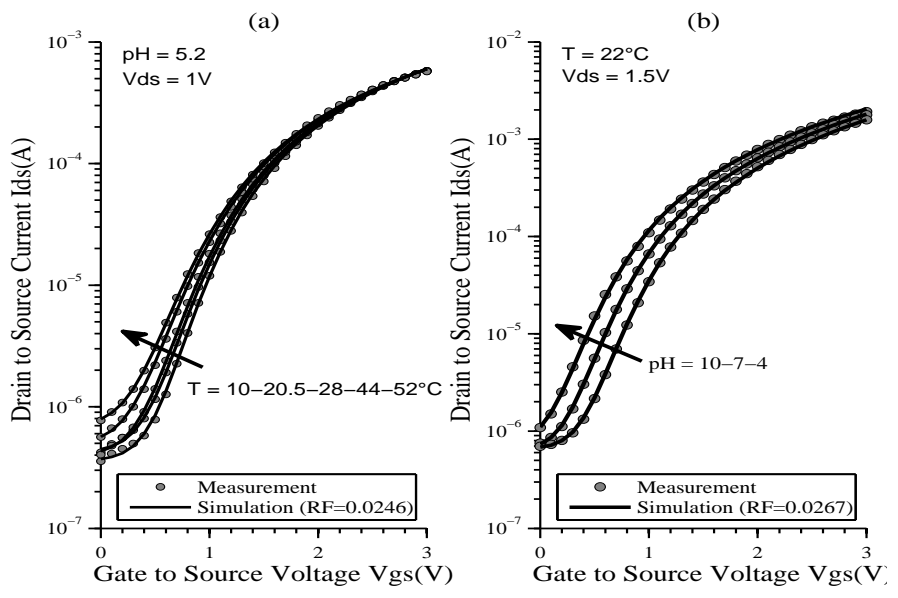

Figure 7: Effect of temperature (a) or $\mathrm{pH}$ (b) variations on Drain-Source current $(I d s)$ vs Gate-Source voltage $(V g s)$ curves. The value of RF indicate the reliability factor of the simulation

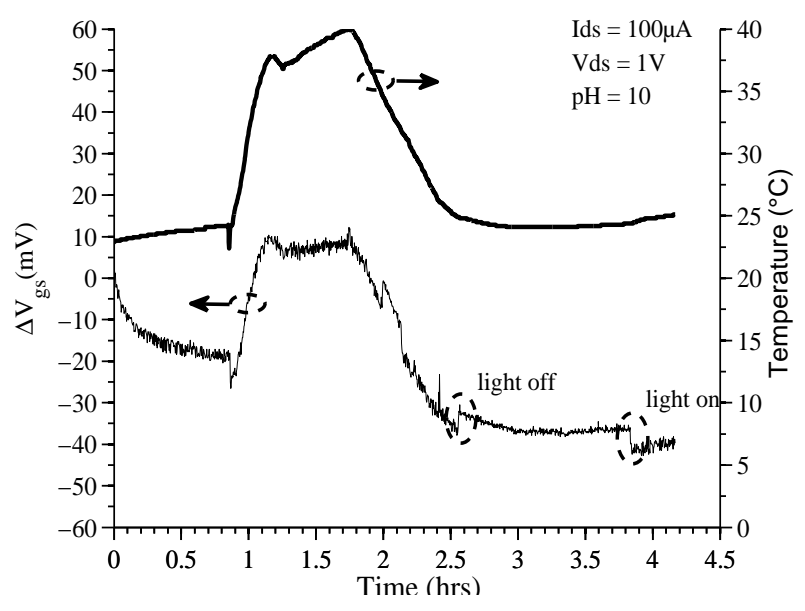

Figure 8: Drift rates of $\mathrm{pH}-\mathrm{ISFET}$ in a solution at $p H=10$ and under an increase of the temperature from $T=22^{\circ} \mathrm{C}$ to around $T=40^{\circ} \mathrm{C}$. The influence of the light switch is shown.

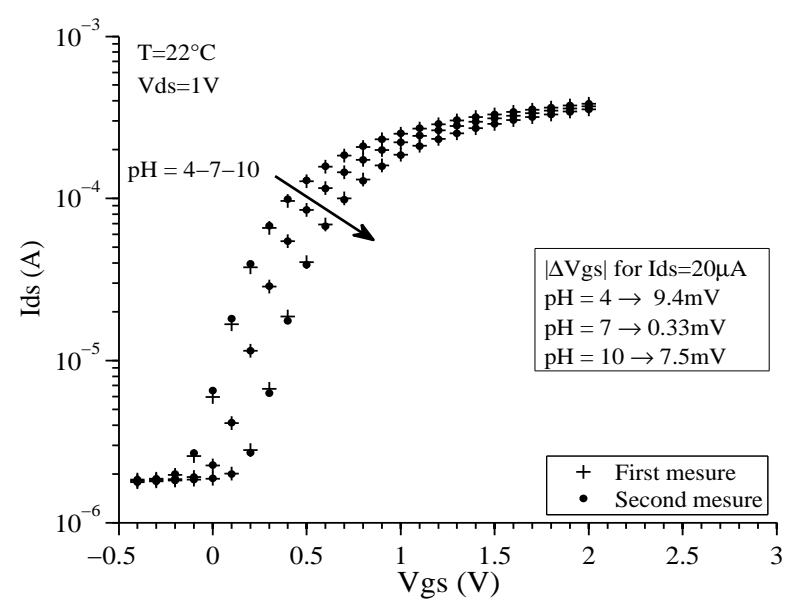

Figure 9: Measured trans-conductance curves for hysteresis cycle of the $\mathrm{pH}$ (4-7-10 $\leftrightarrow 10-7-4)$. 


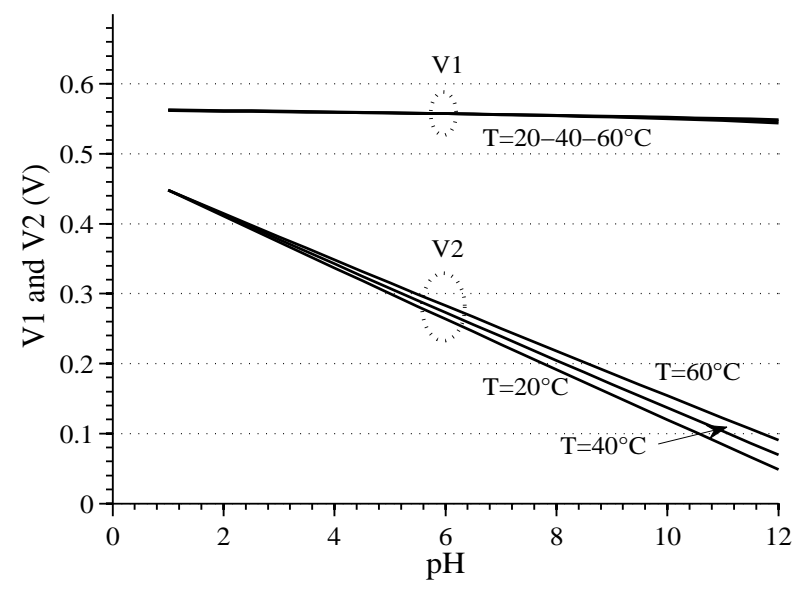

Figure 10: Simulated variation of the potentials $V_{1}$ and $V_{2}$ vs the $\mathrm{pH}$ at $T=$ 20,40 and $60^{\circ} \mathrm{C}$.

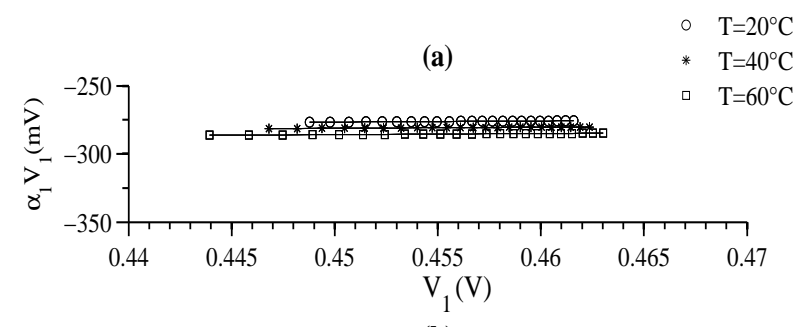

(b)
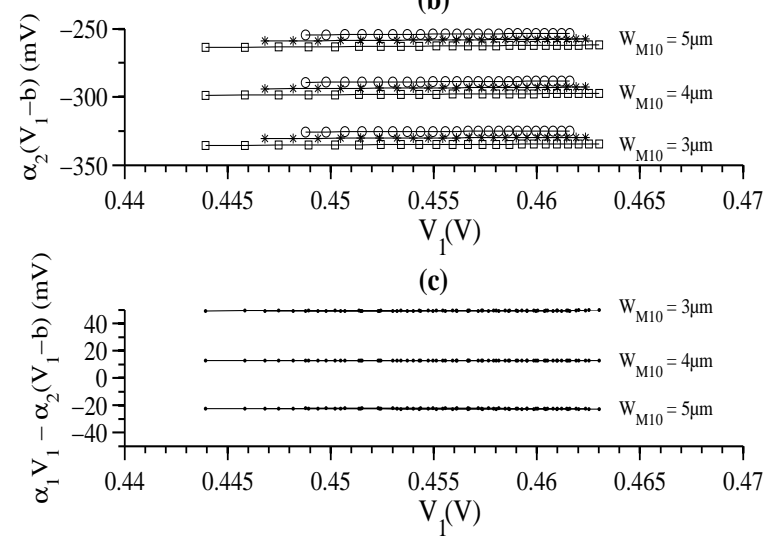

Figure 11: simulations showing the variation of (a) $\alpha_{1} V_{1}$, (b) $\alpha_{2}\left(V_{1}-b\right)$ and (c) $\alpha_{1} V_{1}-\alpha_{2}\left(V_{1}-b\right)$, for $T=20,40$ and $60^{\circ} \mathrm{C}$ and the canal width of the transistor M10, $W_{M 10}=3,4$ and $5 \mu m$, as the potential $V_{1}$ is varied. The interval of variation of $V_{1}$ is deduced from the simulation of the first stage for $\mathrm{pH}$ varying from 2 to 12

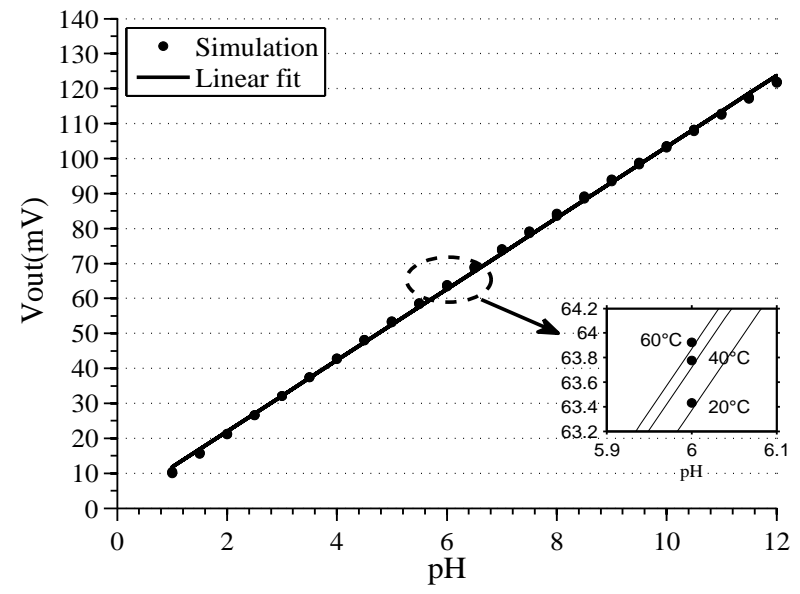

Figure 12: Simulated readout-circuit response for $T=20,40$ and $60^{\circ} C$, the small graph is a zoom on the area around $\mathrm{pH} 6$ to show the very low incidence of the temperature

stage 1. As shown in Fig.11-c, the term $\alpha_{1} V_{1}-\alpha_{2}\left(V_{1}-b\right)$ can be adjusted to a value equal or close to zero by acting on the canal width of the transistor M10. It has to be noted that in Fig.11-c, each of the three curves includes the results of simulations for $T=20,40$ and $60^{\circ} \mathrm{C}$. In the above analysis, the value of the attenuation $\alpha_{1}$ is chosen and then the attenuator circuit sized according to the constraints of stage 3 . The resulting values of $\alpha_{1}$ and $\alpha_{2}$ are around -0.5 and -0.9 respectively.

As illustrated by figure 12, the circuit simulation shows a linear response across a large range of $\mathrm{pH}$, as well as a very insensitive temperature behavior. We show that this compact circuit provides accurate response, although the temperature varies between 20 and $60^{\circ} \mathrm{C}$. The thermal compensation scheme provides an improved thermal stability by eliminating the temperature effect on the circuit output. The width of the transistor M4 of the Caprio's-quad was adjusted to keep the potential $V_{1}$ constant. The simulation results confirm the theoretical considerations presented in the paper. The circuit output presents a maximum voltage drift, with temperature, around $28 \mu \mathrm{V} /{ }^{\circ} \mathrm{C}$ for $\mathrm{pH}=12$ and $3.7 \mu \mathrm{V} /{ }^{\circ} \mathrm{C}$ for $\mathrm{pH}=5$. The maximum power consumption of the readout circuit was estimated to be around $18.6 \mathrm{~mW}$. In integrated IC application perspective, the power supply and the biasing currents can be further minimized by reducing the aspect ratios of ISFET device and all transistors for lowering the total power consumption.

\section{Conclusions}

In this study, we analyzed the behavior of a pH-ISFET sensor shown by changing the temperature and the $\mathrm{pH}$ of the electrolyte solution. The simulation results show that the combination of a simplified EKV model with basic description of a surface potential can fit experimental results with good accuracy in weak and moderate regimes.

The model takes into account a temperature dependency of reference electrode, the $\mathrm{SiO}_{2} / \mathrm{Si}_{3} \mathrm{~N}_{4}$ electrolyte/insulator inter- 
face and the semiconductor part of the pH-ISFET. The model was able to explain the experimental measurements of threshold voltage drift, estimated around $9 \mathrm{mV} / \mathrm{C}$ (at $\mathrm{T}=300 \mathrm{~K}$ ). The extracted value of $p H_{p z c}(4.2$ at $\mathrm{T}=300 \mathrm{~K})$ is in good agreement with the reported values for $S i_{3} N_{4}$ ion sensitive membrane. The temporal drift combined to the thermal drift shows the limits of the $\mathrm{pH}$ sensors, the drift was estimated to $0.02 \mathrm{pH} / \mathrm{h}$. If the thermal hysteresis is performed, this value increase to $0.15 \mathrm{pH} / \mathrm{h}$ for a shift of approximately $20^{\circ} \mathrm{C}$. The study introduces, also, an original architecture that implements a low-power readout circuit for a pH-ISFET featuring thermal compensation, based on MOS transistors operated in sub-threshold. The ISFET thermal model is used to simulate a proposed readout circuit. As a result, the circuit output voltage drift, with temperature, is less than $3.7 \mu \mathrm{V} /{ }^{\circ} \mathrm{C}$, around $\mathrm{pH}=5$. The main advantage of the proposed solution consists in the absence of a temperature sensor, resulting in a greater simplicity of the circuit.

\section{References}

[1] P. Bergveld, Development of an ion-sensitive solid-state device for neurophysiological measurements, IEEE Trans. Biomedical. Eng. BME-17 1 (1970) 70-71.

[2] L. T. Yin, J. C. Chou, W. Y. Chung, T. P. Sun, S. K. Hsiung, Characteristics of silicon nitride after $\mathrm{O}_{2}$ plasma surface treatment for pH-ISFET applications, IEEE Trans. Biomedical Eng. 48 (2001) 340-344.

[3] S. R. Lee, M. M. Rahman, M. Ishida, K. Sawada, Development of a highly-sensitive acetylcholine sensor using a charge-transfer technique on a smart biochip, Trends in Analytical Chemistry. 28 (2009) 196-203.

[4] M. Janicki, M. Daniel, M. Szermer, A. Napieralski, Ion sensitive field effect transistor modelling for multidomain simulation purposes, Microelectronics Journal. 35 (2004) 831-840.

[5] D. Y. Chen, P. K. Chan, An intelligent ISFET sensory system with temperature and drift compensation for long-term monitoring, IEEE Sens. J. 8 (2008) 1948-1959.

[6] S. Jamasb, S. D. Collins, R. L. Smith, A physical model for threshold voltage instability in $\mathrm{Si}_{3} \mathrm{~N}_{4}$-gate $H^{+}$-sensitive FETs (pH ISFETs), IEEE Trans. Elec. Devices. 45 (1998) 1239-1245.

[7] P. K. Chan, D. Y. Chen, A CMOS ISFET interface circuit with dynamic current temperature compensation technique, IEEE Trans. Circuits and Systems. 54 (2007) 119-129.

[8] W. Y. Chunga, Y. T. Lina, D. G. Pijanowskac, C. H. Yangb, M. C. Wanga, A. Krzyskowc, W. Torbiczc, New ISFET interface circuit design with temperature compensation, Microelectronics Journal. 37 (2006) 11051114.

[9] Y. L. China, J. C. Choub, T. P. Sunc, W. Y. Chunga, S. K. Hsiunga, A novel $\mathrm{pH}$ sensitive ISFET with on chip temperature sensing using CMOS standard proces, Sensors and Actuators B. 76 (2001) 582-593.

[10] P. R. Barabash, R. S. C. Cobbold, W. B. Wlodarski, Analysis of the threshold voltage and its temperature dependence in ElectrolyteInsulator-Semiconductor Field-Effect Transistors (EISFET's), IEEE Trans. Elec. Devices. ED-34 (1987) 1271-1282.

[11] L. Shepherda, C. Toumazou, Weak inversion ISFETs for ultra-low power biochemical sensing and real-time analysis, Sensors and Actuators B. 107 (2005) 468-473.

[12] C. C. Enz, F. Krummenacher, E. A. Vittoz, An analytical MOS transistor model valid in all regions of operation and dedicated to low-voltage and low-current applications, Analog Integrated Circuit and Signal Processing. 8 (1995) 83-114.

[13] R. Künhold, H. Ryssel, Modeling the ph response of silicon nitride isfet devices, Sensors and Actuators B. 68 (2000) 307-312.

[14] D. E. Yates, S. Livine, T. W. Healy, Healy, site-binding model of the electrial double layer at the oxide/water interface, J. Chem. Sot., Faraday Trans. I 70 (1974) 1807-1818.

[15] W. M. Siu, R. S. C. Cobbold, Basic properties of the Electrolyte- $\mathrm{SiO}_{2}-\mathrm{Si}$ system: Physical and theoretical aspects, IEEE Trans. Elec. Devices. ED26 (1979) 1805-1815.
[16] D. L. Harame, L. J. Bousse, J. D. Shott, J. Meindl, Ion-sensing devices with silicon nitride and borosilicate glass insulators, IEEE Trans. Elect. Devices. ED-34 (1987) 1700-1707.

[17] C. D. Fung, P. W. Cheung, W. H. Ko, A generalized theory of an electrolyte-insulator-semiconductor field-effect transistor, IEEE Trans. Elect. Devices. ED-33 (1986) 8-18.

[18] S. Martinoia, G. Massobrio, A behavioral macromodel of the ISFET in SPICE, Sensors and Actuators B 62 (2000) 182-189.

[19] J. M. Sallese, M. Bucher, F. Krummenacher, P. Fazan, Inversion charge linearization in MOSFET modeling and rigorous derivation of the EKV compact model, Solid-State Electronics. 47 (2003) 677-683.

[20] M. Bucher, C. Lallement, C. Enz, F. Théodoloz, F. Krummenacher, The EPFL-EKV MOSFET model equations for simulation, EPFL, Lausanne, Switzerland, technical report edition, 1998.

[21] R. S. C. Cobbold, Transducers for Biomedical Measurements: Principles and Applications, Wiley, New York, NY, 1974.

[22] R. Gomer, G. Tryson, An experimental determination of absolute halfcell EMF's and single ion free energies of solvation, J. Chem. Phys. 66 (1977) 4413-4424.

[23] L. Bousse, The chemical sensitivity of Electrolyte/Interface/Silicon structures, PhD Dissertation., Twente University of Technology, 1982.

[24] P. Temple-Boyer, J. Launay, I. Humenyuk, T. Do Conto, A. Martinez, C. Bériet, A. Grisel, Study of front-side connected chemical field effect transistors for water analysis, Microelectronics Reliability 44 (2004) $443-$ 447.

[25] S. Martinoia, G. Massobrio, L. Lorenzelli, Modeling ISFET microsensor and ISFET-based microsystems: a review, Sensors and Actuators B 105 (2005) 14-27.

\section{Biographies}

S.E. Naimi was born on July 23, 1967. He received the DEA degree in microelectronics in 1992 and his $\mathrm{PhD}$ degree from Paul Sabatier University (Toulouse-France) in 1997. He joined STMicroelectronics-Casablanca (Morocco) as product Engineer. He joined the National School of Applied Sciences (ENSA), Mohammed 1st University, in 2001 as a professor. His research activities include the modeling of electrochemical micro-sensors and analog IC design.

B. Hajji was born on 7th December 1970. He received his DEA in microelectronics from the "Université Paul Sabatier" (Toulouse-France) in 1996. He joined the "Laboratoire d'Architecture et d'Analyse des Systèmes" from the French "Centre National de la Recherche Scientifique" in 1996 and received the PhD degree from the "Université Paul Sabatier" in 1999. He joined ST-Microelectronics-Casablanca (Morocco) as product Engineer in 2000 and he jointed the National School of Applied Sciences (Mohammed 1st University) in 2003 as a professor. His research activities and are dedicated to the development of CHEMFETs chemical sensors and embedded system.

I. Humenyuk was born the 29th of July 1977. She received the degree in electronic engineering from the Lviv Polytechnic National University (Ukraine) in 2000 and her "Diplôme d'Etudes Approfondies" in Microelectronics from the "Institut National des Sciences Appliquées de Toulouse" (France) in 2002. She joined the "Laboratoire d'Architecture et d'Analyse des Systèmes" from the French Centre National de la Recherche Scientifique (LAAS-CNRS) in 2002 and received the $\mathrm{PhD}$ degree from the "Institut National des Sciences 
Appliquées de Toulouse" (France) in 2005. She worked on the development of chemical microsensors for water monitoring.

J. Launay was born on March 11, 1975. He received the degree in electronic engineering from the "Institut National des Sciences Appliquées de Toulouse" (France) in 1998. $\mathrm{He}$ joined the "Laboratoire d'Architecture et d'Analyse des Systèmes" from the French "Centre National de la Recherche Scientifique" (LAAS-CNRS) in 1998 and received the $\mathrm{PhD}$ degree from the "Institut National des Sciences Appliquées de Toulouse" (France) in 2001. In 2002, he became lecturer at the University of Toulouse (France). His research activities include the development of electrochemical micro-sensors for the detection in liquid phase.

P. Temple-Boyer was born on October 25, 1966. He received his Engineers Degree in electronic engineering from the "Ecole Supérieure d'Electricité" (Paris-France) in 1990 and his Masters Degree in microelectronics from the University of Toulouse (France) in 1992. He joined the "Laboratoire d'Architecture et d'Analyse des Systèmes" (LAAS) from the French "Centre National de la Recherche Scientifique" (CNRS) in 1992 and received the $\mathrm{PhD}$ degree from the "Institut National des Sciences Appliquées de Toulouse" (France) in 1995. Since then, as a CNRS researcher, he has worked at LAAS on the development of physical and chemical micro-sensors. 